\title{
Point-of-Care Assessment at a Free, Out-of-Hours Orthopedic Clinic
}

\author{
Whitney M. Graves, DAT, LAT, ATC' ; Zachary K. Winkelmann, PhD, SCAT, ATC²; Kenneth E. Games, PhD, \\ LAT, ATCl \\ 'Cumberland University, Lebanon, TN; ${ }^{2}$ University of South Carolina, Columbia, SC; ${ }^{3}$ Indiana State University, Terre Haute, IN
}

\begin{abstract}
Access to healthcare is a challenge for many Americans living in rural communities. Hospital systems have created programs and developed models to improve access to care. However, documentation of the role of these clinics serving different populations and various types of communities is quite scarce. The purpose of this article is to describe the patient satisfaction and procedures assessed at the point-of-care during a 14-week, out-of-hours free orthopedic clinic in the Southeastern United States. One hundred and twenty-nine patients attended the orthopedic clinic and completed a patient satisfaction survey following their patient encounter. At the end of the 14-week, $89 \% \quad(n=115 / 129)$ of the patients were highly satisfied with the staff and physician politeness and $100 \%(n=129 / 129)$ of patient encounters were highly satisfied with staff and physician professional manner. During this study, $72 \%(n=93 / 129)$ of patients reported that their injury and treatment plan was explained in an understandable manner, and $75 \%(n=97 / 129)$ of patient encounters reported that staff and physicians answered all of their questions in an understandable way. Of those surveyed, $95 \%(n=123 / 129)$ of patients reported that they were highly satisfied with the free clinic and the service that was provided. Overall, 96\% ( $n=124 / 129)$ of patients reported that they would come back to the free, out-of-hours orthopedic clinic outside of just the fall football season. The point-of-care assessment described the number of $x$-rays taken, MRI's ordered, follow-up appointments scheduled, and potential revenue generated through surgeries scheduled during the 14-week time period. Moreover, the access to specialized sports medicine care has expanded to reach underserved populations such as pediatrics and those unable to miss work to seek healthcare. The results of this assessment have provided the orthopedic clinic staff and patients with data to support changes to improving patient-centered and low-cost healthcare visits.
\end{abstract}

\section{Key Phrases}

Clinic and Hospital Patient Population, Healthcare Economics (Value and Worth), Organizational and Personal Outcomes

\section{Correspondence}

Dr. Whitney Graves, Indiana State University, 3200 Belotes Ferry Road, Lebanon, TN 37087.

E-mail:wgraves@cumberland.edu

Twitter: @Whit_graves

\section{Full Citation}

Graves WM, Winkelmann ZK, Games KE. Point-of-Care Assessment at a Free, Out-of-Hours Orthopedic Clinic. Clin Pract Athl Train. 2019;2(3):16-21. https://doi.org/10.31622/2019/0003.2

Submitted: March 3, 2019 Accepted: August 7, 2019

\section{INTRODUCTION}

In the United States, a lack of insurance and underinsurance composes a significant national public health problem. ${ }^{1}$ As of 2014 , in the United States, 50.7 million individuals, $16.7 \%$ of the population, were uninsured and many more experienced barriers to obtaining health care. ${ }^{1}$ Some of these barriers include: means/cost of transportation, lack of understanding with medical terminology, and wait time in the waiting room. Despite large gains in health coverage, some people continued to lack coverage, and the Affordable Care Act (ACA) remained the subject of political debate.7 Attempts to repeal and replace the ACA stalled in summer 2017, but there have been several changes to implementation of the ACA under the Trump

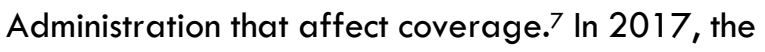
number of uninsured rose for the first time since implementation of the ACA to 27.4 million. $^{7}$ Certain population subgroups have a low amount of health insurance coverage, including ethnic minorities, residents of southern and urban locations, and low-income households. Specifically in the southern state of Alabama, $33 \%$ of its population is uninsured and the highest uninsured rate for the state is in southern Alabama near Birmingham. ${ }^{2}$ There is a substantial need to provide health care for uninsured individuals in the United States and southern states such as Alabama in particular. Previous research has shown that one method to improve access to care is through free medical clinics, which has been cited to have high patient satisfaction feedback 
due to access to care. ${ }^{3}$ At East Alabama Orthopaedics \& Sports Medicine in Opelika, Alabama, the staff provided athletic training services to local secondary schools in the Lee County which includes Auburn, Alabama. Lee County, Alabama has a vast array of current socioeconomic status among residents with the 2016 median household income being \$45,056 and predominantly White residents $(67.9 \%) .{ }^{4}$

In the East Alabama Orthopaedics \& Sports Medicine clinic, the patient panel is predominately lower and middle-class income status, and a high number of self-pay patients. According to the U.S. Census Bureau, the majority of patients seen in the East Alabama Orthopaedics \& Sports Medicine clinic would be considered underserved. Opelika, Alabama is considered a rural area with about $15 \%$ of the population speak a language other than English. There is a 3.5\% Hispanic population and $11.5 \%$ Korean population. About $15 \%$ of the population under the age of 65 has a disability, and about $15-20 \%$ of people under the age of 65 have no form of health insurance. ${ }^{4}$ The poverty rate is at $30 \%$. Twenty-three percent of the population for whom poverty status is determined in Lee County, $\mathrm{AL}(34,085$ out of 148,121 people) live below the poverty line. ${ }^{4}$ This number is higher than the national average of $14 \%$. The largest demographic living in poverty is male between the ages of 18-24, followed by female between the ages of 18-24, and finally female between the ages of 25-35.4 Additionally, the clinic serves an estimated 2,000 veterans from Lee County, Alabama. The cost of healthcare is sometimes a burden on the patients, and especially the studentathletes that the staff provides healthcare to. The student-athletes that we provide medical care make up a large percentage of the underserved community and often times these patients will not come in to see the physician to receive medical treatment due to the office visit or insurance copay costs. At East Alabama Orthopaedics \& Sports Medicine, a patient's continuity of care is a concern as some patients abandon care due to outstanding balances or them not having the funds to come in and see the doctor.

Copyright (C) by Indiana State University
East Alabama Orthopaedics \& Sports Medicine clinic currently has a free orthopedic clinic outside of business hours. The facility serves patients on Saturday mornings outside of the normal clinic hours for 2-4 hours during the fall school semester as it aligns with football season. A typical patient load during one Saturday session is 20 to 25 student-athletes. The free orthopedic clinic runs for 14-weeks beginning the first Saturday following the start of football season and ending the Saturday after the Alabama state championship football game. The services provided at the orthopedic clinic include diagnostic imaging ( $x$ rays), bracing, scheduling of MRls at East Alabama Orthopaedics \& Sports Medicine, full orthopedic evaluations, and concussion assessment. These services are provided and performed at no cost to the student-athlete or their family regardless of insurance status. The staff for the free orthopedic clinic included two orthopedic surgeons, one sports medicine physician with training in neurology, seven athletic trainers, an outreach coordinator, and administrative personnel. The staff was paid as part of their contracts for their services meaning that there were no upfront or recurring costs for stipends or salaries. Currently, the out-of-hours free orthopedic clinic lacks any form of patient survey or satisfaction assessment regarding the services provided from the staff. As such, the purpose of this point-of-care assessment was to explore the patient satisfaction, access to sports medicine services, and potential cost-benefit for the community.

\section{METHODS}

Prior to the start of the 2018 football season, the lead athletic trainer for the free, out-of-hours orthopedic clinic implemented a patient satisfaction survey for patients to complete following their visit. The patient satisfaction survey consisted of five questions measured using 5-point Likert scale with one additional yes/no item. This six-question instrument aimed to determine each patient's perception of the following constructs: (1) politeness and professionalism of personnel, (2) explanation and understanding of injury and 
Table 1. Patient Satisfaction Survey

\section{Question}

Were our personnel polite and courteous?*

Did our personnel take care of you in a professional manner?*

Did we explain your injury and treatment plan in an understandable manner?*

Did we answer all of your questions in an understandable way?*

Overall, how satisfied were you with the free service you received from us?*

Would you come to a Saturday morning clinic outside of football season?\#

* = Measured using Likert scale of 5=Highly Satisfied, 4=Satisfied, 3=Adequate, 2=Unsatisfied, $1=$ Very Unsatisfied, $\#=$ Yes/No item

treatment, (3) satisfaction of the free orthopedic clinic, and (4) if the patient would seek healthcare again from the facility. Table 1 provides the items from the patient satisfaction survey.

Throughout the 14-weeks that the free orthopedic clinic was open, the lead athletic trainer administered a paper survey to each patient as they checked out. The patient satisfaction assessment was collected anonymously. Once the patient completed the survey, the patient placed their survey into a locked drop box near the exit of the East Alabama Orthopaedics \& Sports Medicine clinic. This survey was given and completed by 129 patients. Following the 14 week data collection period, the lead athletic trainer compiled patient responses from the survey using Microsoft Excel. Concurrently, the lead athletic trainer also tracked all patient data and procedures during the office visit that the patient received. The patient data and procedures included the (1) reason for office visit, (2) body region being evaluated, (3) type of evaluation, (3) evaluation complexity, (4) radiographs taken, (5) MRI ordered, (6) surgery scheduled, (7) follow-up appointment scheduled, and (8) if the patient was a football player or non-football player. Upon completion of collecting patient data and procedures, along with the satisfaction surveys, the leader athletic trainer analyzed the data and compiled the findings for a presentation to physicians and East Alabama Orthopaedics \& Sports Medicine stakeholders.

\section{RESULTS}

The study consisted of male and female middle to high school aged patients who participated in football, cheer, volleyball, tennis, and baseball. The major body regions evaluated during the data collection period were knee and shoulder. There were 121 musculoskeletal-based encounters and seven reported concussions. Overall, the majority of evaluation complexity that each physician documented was considered moderate level. Medicare defines an evaluation complexity of moderate level as face to face time spent with a patient requiring 20-25 minutes of care. ${ }^{6}$ Ninety-six radiographs were taken in the clinic and thirty-three patient encounters required an MRI to be ordered. Ninety-four encounters deemed a follow up appointment was necessary and 38 re-check evaluations were documented. Seven surgical procedures were generated during this time frame.

Overall, the results from this study presented a positive effect and an increase in the number of radiographs taken, MRI's ordered, follow-up appointments made, and surgeries each physician obtained during that time period compared to not having a Saturday non- business hours clinic. The results also showed that overall patients were highly satisfied from the healthcare they were 
Table 2. Procedures Performed During the Free Clinic

\begin{tabular}{lccc}
\hline Procedure & Count & Cost & Total Costs Saved \\
\hline Initial evaluation & & & \\
Low & 0 & $\$ 50$ & $\$ 0$ \\
$\quad$ Moderate & 72 & $\$ 75$ & $\$ 5,400$ \\
$\quad$ High & 17 & $\$ 100$ & $\$ 1,700$ \\
Re-Evaluation & & & \\
Low & 0 & $\$ 50$ & $\$ 0$ \\
Moderate & 36 & $\$ 75$ & $\$ 2,700$ \\
High & 2 & $\$ 100$ & $\$ 200$ \\
Radiographs taken & 96 & $\$ 50-\$ 100$ & $\$ 4,800-\$ 9,600$ \\
\hline
\end{tabular}

receiving. The patient satisfaction survey results identified that $89 \%(n=115 / 129)$ of patient encounters were highly satisfied with staff and physician politeness, and $100 \%(n=129 / 129)$ of patients were highly satisfied with the AT and physician professional manner. $95 \%$ $(n=123 / 129)$ of patients reported that they were highly satisfied with the free injury clinic and services that were provided. Overall, 96\% $(n=124 / 129)$ of patients reported that they would come back to a Saturday morning clinic outside of the fall football season.

Moreover, the clinic potentially saved patients anywhere from $\$ 9,400$ to $\$ 14,200$, or $\$ 75$ to $\$ 113$ per patient $(n=125)$. This was based on clinic procedure fees. In addition, the out-of-hours clinic could serve as a revenue stream for East Alabama Orthopaedics \& Sports Medicine through referrals. In order to analyze if the free services performed to potential revenue from follow-up appointments, we calculated the potential revenue using the East Alabama Orthopaedics \& Sports Medicine clinic costs for an MRI, surgical procedure, or a follow-up appointment in the clinic. In total, East Alabama Orthopaedics \& Sports Medicine generated $\$ 32,900$ to $\$ 39,900$ from the 94 patients, or $\$ 350$ to $\$ 424$ per patient. To present this data to stakeholders, a cost-benefit ratio is recommended. For the specific out-of-hours clinic, a total benefit per patient on the low end of procedure fee estimate would be $\$ 350$ and the cost was $\$ 75$ which gives a positive benefit-to-cost ratio of 4.667 .

Copyright $($ b) by Indiana State University
We calculated this cost-benefit ratio by dividing the total benefit per patient (low end- \$350) by the cost (\$75). Table 2 and Table 3 provide details related to the procedures performed during the period, and the follow-up services scheduled with the potential revenue generated for East Alabama Orthopaedics \& Sports Medicine, respectively.

\section{CLINICAL APPLICATION}

Providing patient-centered care is one of the most important things we can do in health care. The patient has options and can choose where to seek services to meet their healthcare needs. Measurement and understanding of the patient, caregiver, and family experience of healthcare provides the opportunity for reflection and improvement of healthcare and patient outcomes. Often times we, as healthcare providers, seem to forget what is really important for patient satisfaction. It is vital to review and reflect on Picker's Eight Principles of Patient-Centered Care in relation to our own practice. ${ }^{5}$ It is important to involve patients in decision making, recognizing they are individuals with their own unique values and preferences. ${ }^{5}$ Proper coordination of care can alleviate patients feeling vulnerable and powerless in the face of an illness or injury ${ }^{5}$ In healthcare, patients expressed their worries that they were not being completely informed about their condition or prognosis. ${ }^{5}$ Physical comfort will come after healthcare providers enhance patient education and information. ${ }^{5}$ The level of physical comfort that patients report has a significant 
Table 3. Potential Revenue for Follow-Up Appointments

\begin{tabular}{lccc}
\hline Procedure & Count & Cost & Potential Revenue \\
\hline MRI & 33 & $\$ 500$ & $\$ 16,500$ \\
Surgical procedure & 7 & $\$ 1,000-\$ 2,000$ & $\$ 7,000-\$ 14,000$ \\
$\begin{array}{l}\text { In Clinic Appointment with } \\
\text { Physician }\end{array}$ & 94 & $\$ 100$ & $\$ 9,400$ \\
\hline
\end{tabular}

impact on their experience with us as providers. Providing accommodation for family and friends and involving them in the decision-making process can enhance patient care and ease the overall patient experience for everyone. Providing patients with understandable information regarding medications, ongoing treatment, physical limitations and access to physical or financial support will supply patients a better continuity and transition to care. Finally, patients need to know they can access care when it is needed. ${ }^{5}$ The ease and availability of scheduling appointments, accessibility to specialists and providing clear instructions on how and when to get referrals are all great examples of how we can enhance patients access to care.

Gaining a better understanding of patient satisfaction, while measuring actual care provided our clinic with greater insight on what areas of patient care need to be strengthened and what areas of patient care we are excelling in. After reflecting on Picker's Eight Principles of Patient Centered Care and our patient satisfaction survey results that was completed in this study, we were pleased to see that our clinic was on the right tract for providing optimal patient centered care. This project has opened opportunities to reach the underserved population, student athletes, and working men and women who cannot get away from their job during the Monday thru Friday work week; and also allow for quality healthcare visits. The result of the cost-benefit ratio demonstrates that the potential revenue generated from $75 \%$ $(n=94 / 125)$ patients (age 18-24) that referred to the East Alabama Orthopaedics \& Sports Medicine clinic for follow-up services will be greater than any "lost" costs incurred by offering the out-of-hours free clinic. As such, the clinic has not only brought a reduced healthcare option to the student-athletes in the county, it also provides a valuable revenue stream to the clinic. In addition, the gains from this project has demonstrated that East Alabama Orthopaedics \& Sports Medicine provides an experience that is highly satisfied by the patients.

After compiling all of the data, the report was presented to East Alabama Orthopaedics \& Sports Medicine stakeholders and physicians. During the presentation, the possibility of extending the free Saturday morning clinics beyond the football season was proposed. During the collaborative discussion, the stakeholders and lead athletic trainer discussed the pros and cons of an extension of providing the out-of-hours clinic during the spring and summer months. After reviewing the results and patient satisfaction responses, the group came to an agreement that the community would greatly benefit from extending the out-of-hour clinic services, while providing East Alabama Orthopaedics \& Sports Medicine an opportunity for community outreach. The increased volume of patients from opening a clinic on Saturday also increases the surgical load and patient visits for physicians, which in turns increases potential revenue to the clinic. We do acknowledge that there are still barriers to care for patients with no insurance regarding follow-up care. Future research should look at different models to continue follow-up patient care for those with no insurance in order to better educate clinics on how to provide the best patient centered care for this population. We suggest that clinics 
providing outreach and per diem athletic training services consider creating an out-of-hours clinic using a similar model to improve the access to care for the population, which improves the chance that patients come back for further care in turn generating in increased revenue stream.

\section{REFERENCES}

1. Rebholz CM, Macomber MW, Althoff MD, et al. Integrated models of education and service involving community-based health care for underserved populations: Tulane student-run free clinics. Southern Med J.

2013;106(3):217-223. https://doi.org/10.1097/SMJ.0b013e3182 87fe9a.

2. Fronstin P. Sources of health insurance and characteristics of the uninsured: Analysis of the March 2011 current population survey. EBRI Issue Brief. $2011 ; 362$.

3. Ellett JD, Campbell JA, Gonsalves WC. Patient satisfaction in a student-run free medical clinic. Fam Med J. 2010;42(1):6-18.

4. Data USA. Data USA: Lee County, AL. https://datausa.io/profile/geo/lee-countyal/. Accessed February 25, 2019.

5. Oneview. The Eight Principles of PatientCentered Care.

https://www.oneviewhealthcare.com/theeight-principles-of-patient-centered-care/.

6. Accessed February 26, 2019.

7. Medicare Learning Network: Documentation Guidelines for Evaluation and Management (E/M) Services.

https://www.cms.gov/Outreach-andEducation/MedicareLearningNetworkMLN/MLNEdWebGuide/E MDOC.html. Accessed April 30, 2019.

8. The Uninsured and the ACA: A Primer - Key Facts about Health Insurance and the Uninsured amidst Changes to the Affordable Care Act.

https://www.kff.org/uninsured/report/theuninsured-and-the-aca-a-primer-key-facts- about-health-insurance-and-the-uninsuredamidst-changes-to-the-affordable-care-act/. Accessed April 30, 2019. 